\title{
Contrôle de la formation osseuse par l'axe hypothalamique : une nouvelle fonction de la leptine
}

D ans l'espèce humaine, de la fin de la puberté à l'arrêt des fonctions gonadiques, la masse osseuse ne varie que très peu et ce grâce à un équilibre entre la production de matrice osseuse par les ostéoblastes, et sa résorption par les ostéoclastes. C'est cet équilibre qui est rompu dans l'ostéoporose, au profit d'une résorption accrue, qui fragilise les os. Cause importante de morbidité dans les pays industrialisés, particulièrement chez les femmes après la ménopause, la perte osseuse qui caractérise l'ostéoporose est difficilement réparée, essentiellement parce que nous connaissons très mal les mécanismes moléculaires contrôlant la formation osseuse [1]. Une étude de notre équipe du Baylor College of Medecine (Houston, TX, USA), qui vient de paraître dans Cell [2], démontre que la production de la matrice osseuse par les ostéoblastes pourrait être sous le contrôle de la leptine. Cela suggère donc que, comme c'est le cas pour le processus de résorption osseuse [3], la fonction des ostéoblastes serait, elle aussi, sous un contrôle de type endocrinien mettant en jeu des molécules circulantes plutôt qu'une régulation tissulaire locale de type autocrine ou paracrine.

La perte osseuse qui survient après la ménopause est une conséquence de l'hypogonadisme; l'obésité protège d'une telle perte osseuse. C'est en se basant sur ces observations cliniques simples que nous avons fait l'hypothèse qu'un mécanisme moléculaire ou endocrinien commun contrôlerait la masse osseuse, le poids et la fonction gonadique. La leptine a été immédiatement suspectée parce que cette hormone polypeptidique secrétée principalement par l'adipocyte contrôle l'homéostasie pondérale et la fonction gonadique par l'intermédiaire de récepteurs hypothalamiques $\left([4,5], m / s 1998, n^{\circ} 5\right.$, p. 675 ; 1999, $n^{\circ} 11$, p. 1276). Pour faire la preuve de sa responsabilité, nous avons utilisé deux souches de souris qui ont la particularité d'être à la fois obèses et stériles: les souris ob/ob [6], dépourvues de leptine, et les souris $\mathrm{db} / \mathrm{db}$ knock-out pour le gène codant pour le récepteur de la leptine [7] $\left(\mathrm{m} / \mathrm{s}\right.$ 1996, $\left.n^{\circ} 3, p .386\right)$. L'étude histomorphométrique des os des souris homozygotes ob/ob et $\mathrm{db} / \mathrm{db}$ a révélé une masse osseuse deux fois plus élevée que celle des souris sauvages. Ce résultat était très inattendu: en effet, l'hypofonctionnement gonadique entraîne toujours une augmentation de la résorption osseuse et, qui plus est, l'hypercortisolisme de ces souris aurait dû aggraver la résorption osseuse. Ces souris représentaient donc un modèle exceptionnel (le seul) où coexistaient une insuffisance de la fonction gonadique et un accroissement de la masse osseuse. L'altération du métabolisme osseux n'était pas secondaire à l'obésité mais bien directement la conséquence de l'absence de leptine: en effet, l'augmentation de la masse osseuse est aussi détectable chez les souris hétérozygotes $(\mathrm{ob} /+)$, qui ne sont pas obèses, et chez les souris homozygotes (ob/ob) lorsqu'on les soumet à un régime alimentaire pauvre en graisses.

L'augmentation de la masse osseuse chez les souris mutantes est liée à une augmentation de la formation osseuse par les ostéoblastes. Celle-ci n'intervient pas par le biais d'une augmentation du nombre des ostéoblastes, qui reste comparable à celui des souris sauvages. En revanche, la quantité de matrice osseuse produite par chaque ostéoblaste est multipliée par deux voire par trois, selon l'âge de la souris. Il n'y a aucun déficit de la fonction des ostéoclastes. L'absence de leptine stimule donc la fonction des ostéoblastes, entraînant une augmentation de la masse osseuse, et ceci malgré l'augmentation de la résorption osseuse liée à l'hypogonadisme. On peut faire un parallèle entre le mode d'action de la leptine sur les ostéoblastes et celui d'une autre molécule très médiatisée récemment, l'ostéoprotégérine (OPG), qui, elle, inhibe la fonction des ostéoclastes lorsqu'elle se lie à son ligand l'OPG-L ([3] et $\mathrm{m} / \mathrm{s} 1999$, $n^{\circ} 8-9, p$. 990), et qui, comme la leptine, est une protéine circulante.

Toutefois, l'action de la leptine est indirecte: en effet, les ostéoblastes n'expriment pas l'isoforme active du récepteur de la leptine (Ob-Rb), dont l'expression est essentiellement restreinte à l'hypothalamus. De plus, la leptine, lorsqu'elle est ajoutée à des ostéoblastes en culture, n'active, dans ces cellules, aucun des signaux de la cascade de transduction $(\mathrm{m} / \mathrm{s}$ 1997, $n^{\circ} 1$, p. 99). Enfin, argument supplémentaire, les ostéoblastes isolés à partir de tissus de souris sauvages ou de souris $\mathrm{db} / \mathrm{db}$ (n'exprimant pas le récepteur de la leptine) synthétisent et minéralisent la matrice extracellulaire avec la même efficacité. Puisque la cellule cible de la leptine n'était pas l'ostéoblaste, on pouvait penser qu'elle agissait via ses récepteurs hypothalamiques. Afin de confirmer cette hypothèse, nous avons traité des souris ob/ob et des souris sauvages pendant un mois par de la leptine, injectée directement dans le troisième ventricule cérébral par l'intermédiaire d'une pompe. La 
masse osseuse des souris ob/ob s'est normalisée tandis que les souris sauvages, dont la masse osseuse était initialement normale, ont développé une ostéoporose.

Tous ces résultats suggèrent donc que le remodelage osseux est sous le contrôle de l'hypothalamus, et que cette régulation centrale aurait une importance fonctionnelle supérieure à celle des autres mécanismes de régulation connus. En effet, seule cette régulation peut contrebalancer les effets délétères de l'hypogonadisme sur la masse osseuse. L'implication obligée de ce nouveau paradigme biologique est que l'ostéoporose, la plus fréquente des altérations du remodelage osseux, aurait aussi une composante centrale. Il reste maintenant à identifier les intermédiaires entre l'hypothalamus et l'ostéoblaste. On peut souligner à ce propos que le neuropeptide Y $\left(\mathrm{m} / \mathrm{s} 1998, n^{\circ} 4\right.$, p. 496) n'antagonise pas la fonction de la leptine dans le remodelage osseux. Nul doute cependant que de ces études émanent de nouvelles perspectives thérapeutiques pour le traitement de l'ostéoporose dont l'objectif serait non plus de limiter la résorption mais aussi de stimuler la formation de matrice osseuse. La recherche d'inhibiteurs de la leptine capables d'agir sélectivement sur la fonction osseuse, sans induire d'obésité, est à l'évidence le premier axe de recherches à explorer. Enfin, la leptine étant un inhibiteur de la formation osseuse, pourquoi ne pas envisager aussi l'existence d'un activateur de la formation osseuse?

\section{RÉFÉRENCES}

1. Karsenty G. The genetic transformation of bone biology. Genes Dev 1999; 13: 303751

2. Ducy P, Amling M, Takeda S, et al. Leptin inhibits bone formation through an hypothalamic relay: a central control of bone mass. Cell 2000; 100: 197-207.

3. Simonet WS, Lacey DL, Dunstan CR, et al. Osteoprotegerin: a novel secreted protein involved in the regulation of bone density. Cell 1997; 89: 309-19.

4. Friedman JM, Halaas JL. Leptin and the regulation of body weight in mammals. Nature 1998; 395 : 763-70.
5. Bruneau G, Vaisse C, Caraty A, Monget P. La leptine: une clef pour la reproduction. Med Sci 1999; 15: 191-6.

6. Zhang Y, Proenca R, Maffei M, Barone M, Leopold L, Friedman JM. Positional cloning of the mouse obese gene and its human homologue. Nature 1994; 372: 425-32.

7. Tartaglia LA, Dembski M, Weng X, et al. Identification and expression cloning of a leptin receptor, OB-R. Cell 1995; 83 : 126371 .

\section{Gérard Karsenty Patricia Ducy}

Department of Molecular and Human Genetics, Baylor College of Medecine, Houston, Texas 77030, Etats-Unis.

\section{TIRÉS À PART}

G. Karsenty.

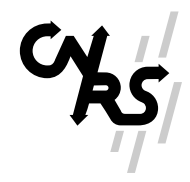

